\title{
Akzeptanz des Triple P-Elterngruppentrainings im Rahmen der kinderpsychiatrischen Behandlung
}

\author{
Wolfgang Briegel ${ }^{\mathrm{a}} \quad$ Ann-Katrin Job ${ }^{\mathrm{b}} \quad$ Kurt Hahlweg ${ }^{\mathrm{b}}$ \\ ${ }^{a}$ Klinik für Kinder- und Jugendpsychiatrie, Psychosomatik und Psychotherapie, Leopoldina-Krankenhaus, Schweinfurt, \\ ${ }^{\mathrm{b}}$ Institut für Psychologie, Technische Universität Braunschweig, Deutschland
}

\section{Schlüsselwörter}

Elterntraining · Triple P · Kinderpsychiatrie

\section{Zusammenfassung}

Hintergrund: Untersucht wurde die Akzeptanz eines zeitlich adaptierten Triple P-Elterngruppentrainings im Rahmen der kinderpsychiatrischen Behandlung. Methodik: 39 Mütter und 14 Väter von 40 Kindern im Alter von 4-11 Jahren, die überwiegend eine expansive Störung aufwiesen, nahmen an der Studie teil. 21 Familien wiesen einen niedrigen sozioökonomischen Status auf, nur 2 einen hohen. 22 Kinder befanden sich zu Beginn des jeweiligen Elternkurses in ambulanter, 14 in tagesklinischer und 4 in stationärer Behandlung. Die Zufriedenheit der Eltern nach Kursende wurde mit einer deutschen Version des "Client Satisfaction Questionnaire» (CSO) erfasst. Mit einem eigens konzipierten Fragebogen wurde 6 Monate nach Trainingsende untersucht, wie häufig die Eltern die vermittelten Strategien noch einsetzten. Ergebnisse: 39 der 40 Familien beendeten das Elterntraining regulär, von diesen bewerteten alle das Programm als gut bis hervorragend. Es fanden sich für die Kurszufriedenheit keine signifikanten Unterschiede in Abhängigkeit vom sozioökonomischen Status, dem Behandlungssetting oder der ICD-10-Diagnose. Bei einer Dropout-Rate von 22,6\% 6 Monate nach Kursende setzten noch $90 \%$ der Eltern etwa die Hälfte der vermittelten Erziehungsstrategien gelegentlich bis regelmäßig ein. Schlussfolgerungen: Ein zeitlich adaptiertes Triple P-Elterngruppentraining wird im Rahmen der kinderpsychiatrischen Behandlung gut angenommen, auch von Familien mit niedrigem sozioökonomischen Status.

\section{Keywords}

Parenting program - Triple P . Child psychiatry

\section{Summary \\ Acceptance of Group Triple P in a Child Psychiatry Setting}

Background: We examined the acceptance of and satisfaction with a time-adapted form of the Triple $P$ parenting program in psychiatric treatment for children. Methods: 39 mothers and 14 fathers from 40 families with children aged 4-11 years with predominantly expansive disorders participated in this study. Socioeconomic status was low in 21 families and high in only 2 cases. At the beginning of the parent group training 22 children were outpatients, 14 day clinic patients and 4 inpatients. Satisfaction with the training was measured by a German version of the Client Satisfaction Questionnaire (CSO). With a specially developed questionnaire parents were asked about the frequency with which they still used Triple P strategies 6 months after training. Results: 39 out of 40 families completed the training. All parents evaluated the group program from good to excellent. No significant differences could be found for program satisfaction depending on socioeconomic status, treatment setting or ICD-10 diagnosis. At 6-month follow-up (dropout rate: $22.6 \%$ ), $90 \%$ of the parents used about half of the Triple P strategies occasionally to regularly. Conclusions: A time-adapted form of the Triple P group training seems to be highly acceptable as a parent-focused intervention in a child psychiatry setting, independent of socioeconomic status.

\section{KARGER \\ Fax +497614520714 \\ Information@Karger.com}

www.karger.com
(C) 2013 S. Karger GmbH, Freiburg

$1016-6262 / 13 / 0234-0252 \$ 38.00 / 0$

Accessible online at:

www.karger.com/ver
PD Dr. med. Wolfgang Briege

Klinik für Kinder- und Jugendpsychiatrie, Psychosomatik und Psychotherapie

Gustav-Adolf-Straße 4, 97422, Schweinfurt, Deutschland

wbriegel@leopoldina.de 


\section{Einleitung}

Therapeutische Interventionen, die sich in randomisierten kontrollierten Studien als wirksam erwiesen haben, lassen sich nicht zwangsläufig im klinischen Alltag genauso erfolgreich einsetzen. Wie gut der Transfer von der Forschung in die Praxis gelingt, hängt unter anderem von der Akzeptanz einer Intervention durch ihre Teilnehmer ab.

Das von Sanders und Mitarbeitern in Australien entwickelte «Positive Parenting Program» (Triple P oder PPP) [Sanders, 2012] stellt ein auf die individuellen Bedürfnisse von Eltern zugeschnittenes 5-Ebenen-Programm dar, das von universeller Prävention (Ebene 1) bis zu Intervention (Ebenen 4 und 5) reicht und auf den Grundprinzipien Suffizienz, Subsidiarität und Selbstregulation beruht.

Für das Triple P liegen trotz seiner guten Evidenzbasierung für die Behandlung expansiver kindlicher Störungen [Eyberg et al., 2008] bis dato keine Untersuchungen zum Einsatz im kinderpsychiatrischen Setting vor, wo Elterntrainings insbesondere bei expansiven kindlichen Störungen eine große Bedeutung zukommt [Bachmann et al., 2008]. Die vorliegende Pilotstudie hatte daher zum Ziel, anhand von Teilnahmerate, Kurszufriedenheit der Teilnehmer sowie Akzeptanz der Erziehungsstrategien zu erfassen, wie das Triple P-Elterngruppentraining im klinischen Alltag der kinderpsychiatrischen Behandlung, die durch Multimodalität gekennzeichnet ist, angenommen wird. Dabei sollte auch untersucht werden, ob Kurszufriedenheit und Akzeptanz in Anhängigkeit vom soziö̈konomischen Status der Eltern, dem Behandlungssetting (ambulant vs. (teil-)stationär) oder der ICD-10-Diagnose des Kindes (Störung des Sozialverhaltens vs. keine Störung des Sozialverhaltens) variieren. Da Kinder aus Familien mit niedrigem sozioökonomischen Status häufiger psychische Probleme und Verhaltensauffälligkeiten zeigen [RobertKoch-Institut und Bundeszentrale für gesundheitliche Aufklärung, 2008], sollte eine therapeutische Intervention idealerweise auch von Eltern mit geringem sozioökonomischen Status gut angenommen werden. Ähnliches sollte für Familien gelten, bei denen eine ambulante Behandlung nicht ausreichend ist oder bei denen das Kind eine Störung des Sozialverhaltens (SSV) aufweist, die bekanntermaßen bei frühem Beginn mit einer ungünstigen Prognose verbunden ist [Stadler, 2012]. A priori wurde von keinen Unterschieden ausgegangen.

\section{Methode}

\section{Durchführung}

Die Datenerhebung fand von Oktober 2008 bis Februar 2010 in der Klinik für Kinder- und Jugendpsychiatrie, Psychosomatik und Psychotherapie am Leopoldina-Krankenhaus der Stadt Schweinfurt statt. Die Teilnahme an der Pilotstudie war freiwillig und wurde, unabhängig vom Behandlungssetting (ambulant, (teil-)stationär), allen Eltern angeboten, die mit ihrem 4-11 Jahre alten Kind eine umfangreiche Diagnostik gemäß den aktuellen Leitlinien der kinder- und jugendpsychiatrischen Fachgesellschaften [Deutsche Gesellschaft für Kinder- und Jugendpsychiatrie und Psychotherapie et al., 2007] durchlaufen hatten und nach klinischer Einschätzung ein Elterntraining benötigten. Die diagnostische Abklärung umfasste 4-5 Termine; dabei wurden folgende Erhebungen regelhaft durchgeführt: Anamnese, psychopathologischer Befund (nach dem Psychopathologischen Befund-System für Kinder und Jugendliche (CASCAP-D) [Döpfner et al., 1999]), Intelligenztestung mittels HannoverWechsler-Intelligenztest für das Vorschulalter (HAWIVA-III [Ricken et al., 2007]) oder Hamburg-Wechsler-Intelligenztest für Kinder (anfangs HAWIK-III [Tewes et al., 2002], später HAWIK-IV [Petermann und Petermann, 2008]), standardisierte störungsspezifische Fremd-, altersabhängig auch Selbstbeurteilungen mittels Diagnostik-System für psychische Störungen nach ICD-10 und DSM-IV für Kinder und Jugendliche-II (DISYPS-II [Döpfner et al., 2008]), Aufmerksamkeitsdiagnostik mittels Testbatterie zur Aufmerksamkeitsprüfung (TAP [Zimmermann und Fimm, 2007]), Verhaltensbeobachtungen und Telefonate mit Kindergarten/Schule zum Zweck der Fremdanamnese. Bei klinischen Hinweisen erfolgte eine Teilleistungsdiagnostik (logopädische Abklärung bei Verdacht auf Sprachentwicklungsverzögerung; Einsatz üblicher standardisierter Testverfahren bei Verdacht auf schulische Teilleistungsstörungen, z.B. Deutscher Mathematiktest für zweite Klassen (DEMAT 2+ [Krajewski et al., 2004]), Diagnostischer Rechtschreibtest für zweite Klassen (DRT 2 [Müller, 2004]), Zürcher Lesetest (ZLT [Linder und Grissemann, 2000])). Bedarf für ein Elterntraining wurde bei deutlichen Eltern-KindInteraktionsproblemen sowie pädagogischen Defiziten der Eltern gesehen. Die Platzvergabe für das Elterngruppentraining erfolgte chronologisch nach Eingang der Anmeldung bzw. des schriftlichen Einverständnisses.

Als Ausschlusskriterien wurde für die Eltern Folgendes definiert: geistige Behinderung (Einschätzung gemäß klinischem Eindruck unter Berücksichtigung des Schulabschlusses), schwere psychische Störung, mangelnde Gruppenfähigkeit oder unzureichende Sprachkenntnisse (jeweils gemäß klinischem Eindruck).

Inhalt und Ablauf des Triple P-Elterngruppentrainings (Ebene 4) richteten sich strikt nach dem aktuellen Gruppenarbeitsbuch (Tab. 1 [Markie-Dadds et al., 2007]). Da von zahlreichen Eltern, die an früheren Triple P-Elterngruppentrainings in der Klinik teilgenommen hatten, mehr Zeit für Übungen angeregt worden war, wurde a priori für alle Sitzungen, insbesondere aber die besonders übungsintensive Sitzung III, mehr Zeit veranschlagt: 150 min für Gruppensitzung I und IV sowie die Abschlusssitzung, 180 min für Sitzung II und 240 min für Sitzung III (empfohlene Zeit im Original-Elterngruppentraining: jeweils 120-135 min).

Da die Untersuchung als Pilotstudie konzipiert war, wurde vorab eine Teilnehmerzahl von 40 Familien festgelegt. Eltern, die trotz klinischen Bedarfs nicht an der Pilotstudie teilnehmen konnten, wurden auch nach Studienabschluss Triple P-Elterngruppentrainings angeboten. Das Versuchsprotokoll wurde von der zuständigen Ethikkommission genehmigt.

\section{Messinstrumente}

Zwecks besserer Vergleichbarkeit wurde die Zufriedenheit der Eltern mit dem Triple P-Elterngruppentraining direkt nach dessen Abschluss mit der deutschen Version des «Client Satisfaction Questionnaire» (CSQ) [Turner et al., 2002] erfasst, welche in zahlreichen Voruntersuchungen zu Triple P Anwendung fand, obwohl es bis dato keine deutsche Normierung gibt. Bei insgesamt 13 Fragen, die auf einer 7-Punkte-Skala beantwortet werden, kann der Gesamtwert 13-91 Punkte betragen. Es gilt: je höher die Punktzahl, desto besser die Bewertung (z.B. «Wie zufrieden sind Sie mit den bisherigen Fortschritten Ihres Kindes?» 1 = sehr unzufrieden; 2 = unzufrieden; 3 = etwas unzufrieden; 4 = neutral; $5=$ eher zufrieden; 6 = zufrieden; $7=$ sehr zufrieden). Die interne Konsistenz des CSQ lag in der vorliegende Stichprobe für die Mütter bei Cronbachs $\alpha=$ 0,94 und für die Väter bei $\alpha=0,95$.

Sechs Monate nach Trainingsende wurde mittels des eigens konzipierten «Fragebogen zur Anwendung der vermittelten Erziehungsstrategien» im Alltag erfasst, wie häufig die Eltern die insgesamt 17 im Kurs vermittelten Strategien noch einsetzten (Frage: «Welche der folgenden 
Tab. 1. Die

Inhalte der Triple

P-Gruppensitzungen

[Markie-Dadds et al., 2007]

\begin{tabular}{|c|c|}
\hline Gruppensitzung & Inhalte \\
\hline I. Positive Erziehung & $\begin{array}{l}\text { was bedeutet «positive Erziehung?» } \\
\text { Faktoren, die das Verhalten von Kindern beeinflussen } \\
\text { Ziele für Veränderungen } \\
\text { das Verhalten des Kindes systematisch beobachten }\end{array}$ \\
\hline $\begin{array}{l}\text { II. Die Entwicklung des } \\
\text { Kindes fördern }\end{array}$ & $\begin{array}{l}\text { eine gute Beziehung zu Ihrem Kind fördern und stärken } \\
\text { wertvolle Zeit mit Ihrem Kind verbringen } \\
\text { mit Ihrem Kind reden } \\
\text { Zuneigung zeigen } \\
\text { angemessenes Verhalten fördern } \\
\text { Ihr Kind loben } \\
\text { Aufmerksamkeit schenken } \\
\text { für anregende Beschäftigungen sorgen } \\
\text { neue Fertigkeiten und Verhaltensweisen beibringen } \\
\text { ein gutes Vorbild sein } \\
\text { beiläufiges Lernen nutzen } \\
\text { Fragen-Sagen-Tun anwenden } \\
\text { eine Punktekarte einsetzen }\end{array}$ \\
\hline $\begin{array}{l}\text { III. Mit Problemverhalten } \\
\text { umgehen }\end{array}$ & $\begin{array}{l}\text { klare Familienregeln aufstellen } \\
\text { mit direktem Ansprechen auf die Nichtbeachtung von Regeln reagieren } \\
\text { bei leichtem Problemverhalten absichtliches Ignorieren einsetzen } \\
\text { klare, ruhige Anweisungen geben } \\
\text { Anweisungen mit logischen Konsequenzen untermauern } \\
\text { stille Zeit einsetzen, um mit Problemverhalten umzugehen } \\
\text { die Auszeit für schwerwiegenderes Problemverhalten einsetzen } \\
\text { Erziehungsroutinen entwickeln } \\
\text { die Vorbereitungen für den Einsatz der Punktekarte abschließen }\end{array}$ \\
\hline IV. Vorausplanen & $\begin{array}{l}\text { Überlebenstipps für Familien } \\
\text { Risikosituationen } \\
\text { Aktivitätenpläne } \\
\text { die Telefonsitzungen vorbereiten }\end{array}$ \\
\hline V-VIII. Abschlusssitzung & $\begin{array}{l}\text { das Programm beenden } \\
\text { Bilanz ziehen } \\
\text { Veränderungen aufrechterhalten } \\
\text { zukünftige Probleme lösen } \\
\text { zukünftige Ziele }\end{array}$ \\
\hline
\end{tabular}

Triple P-Strategien wenden Sie aktuell wie oft an?»). Die Beantwortung für jede einzelne Strategie (z.B. «Punktekarte») erfolgte anhand folgender 5 Antwortmöglichkeiten: «nie», «selten», «gelegentlich», «häufig», «regelmäßig». Die interne Konsistenz dieses Fragebogens lag für die Mütter der vorliegenden Stichprobe bei $\alpha=0,87$ und für die Väter bei $\alpha=0,85$.

Sämtliche Fragebögen wurden nicht durch die Trainer, sondern über das Sekretariat der Klinik ausgegeben und dort auch auf Vollständigkeit kontrolliert.

\section{Stichprobe}

Im Zeitraum vom 01.01.2008 bis 31.01.2010 wurden insgesamt 173 Kinder im Alter von 4-11 Jahren erstmalig in der Schweinfurter Klinik vorgestellt. Von den Familien, die die oben genannten Einschlusskriterien erfüllten, wurden die ersten 40 in die Studie aufgenommen. Insgesamt gingen sukzessive 5 Trainings (jeweils 8 Familien pro Gruppe; gemischte Gruppen aus Eltern von Kindern in tagesklinischer, stationärer und ambulanter Behandlung) in die Studie ein; die Sitzungsdauer betrug im Mittel 180 (Spannweite: 135-245, Standardabweichung (SD): 39 min. Ein Kurs erstreckte sich im Mittel über 10 Wochen; er wurde jeweils von 2 lizensierten Trainern durchgeführt.

Am Elterngruppentraining nahmen insgesamt 39 Mütter und 14 Väter teil. Das Alter der Mütter betrug 26-55 Jahre (Mittelwert (M): 38,5; SD: 6,3), das der Väter 31-52 Jahre (M: 40,5; SD: 5,9). 29 Familien waren
2-Eltern-Familien, wobei in 13 Fällen beide Eltern am Training teilnahmen. Der analog der Studie zur Gesundheit von Kindern und Jugendlichen in Deutschland (KiGGS) ermittelte sozioökonomische Status [Lange et al., 2007] war bei 21 Familien niedrig (53\%), bei 17 mittel (43\%) und bei 2 hoch (4\%).

Von den 40 Index-Kindern waren 29 männlich, das Alter betrug 4-11 Jahre (M: 8,6; SD: 1,6). Als Achse-I-Hauptdiagnose nach ICD-10 wiesen 17 Kinder (43\%) eine einfache Aktivitäts- und Aufmerksamkeitsstörung auf, 15 eine (hyperkinetische) Störung des Sozialverhaltens (38\%), 4 eine Aufmerksamkeitsstörung ohne Hyperaktivität, 2 einen elektiven Mutismus und je ein Kind eine emotionale Störung des Kindesalters mit Geschwisterrivalität bzw. mit sozialer Überängstlichkeit. Zusätzlich lag bei 18 Patienten (45\%) mindestens eine weitere Achse-I-Diagnose und bei 26 (65\%) eine Achse-II-Diagnose vor. $43 \%$ der Kinder zeigten auf der Achse VI zumindest eine ernsthafte und durchgängige soziale Beeinträchtigung in den meisten Bereichen. Zu Beginn des jeweiligen Elternkurses befanden sich 22 Kinder in ambulanter, 14 in tagesklinischer und 4 in stationärer Behandlung. Psychopharmakologisch wurden zu Kursbeginn 30 Kinder behandelt (überwiegend mit Methylphenidat-Präparaten).

\section{Statistische Analysen}

Die Datenanalyse erfolgte mit dem Programm IBM SPSS Statistics 19. Unterschiede zwischen Gruppen (Mütter vs. Väter; niedriger vs. mittlerer 
Abb. 1. Mittlere Bewertung der Triple P-Gruppentrainings durch die Eltern.

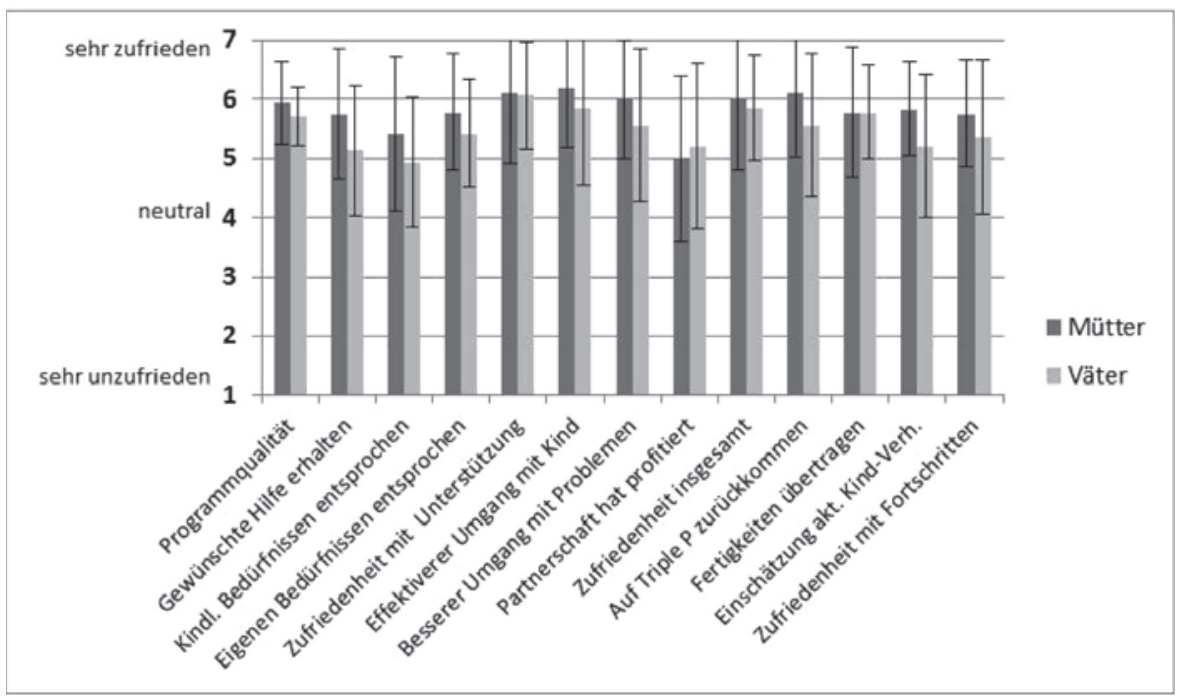

vs. hoher sozioökonomischer Status; ambulante vs. (teil-)stationäre Behandlung; Vorliegen oder Fehlen einer SSV) wurden aufgrund der geringen Stichprobengrößen mit Hilfe des nonparametrischen Mann-WhitneyU-Tests überprüft, statistische Signifikanz wurde als $\mathrm{p} \leq 0,05$ (zweiseitig) definiert. Als Maß für die Größe signifikanter Unterschiede wurden darüber hinaus Intergruppen-Effektstärken berechnet. Verwendet wurde dazu die Formel $\mathrm{d}=\left(\mathrm{M}_{\text {Gruppe1 }}-\mathrm{M}_{\text {Gruppe } 2}\right) /$ Standardabweichung der Differenz nach Rustenbach [2003, S. 81]. Hierbei gelten Effektstärken von 0,20-0,49 als niedrig, von 0,50-0,79 als moderat und ab 0,80 als hoch [Cohen, 1988].

\section{Ergebnisse}

\section{Teilnahmerate}

39 der 40 Familien beendeten das Elterngruppentraining regulär (Drop-out-Rate: $1,9 \%$ ), d.h. sie nahmen an mindestens 3 der ersten 4 Gruppensitzungen, mindestens einem Telefontermin bzw. dem Abschlusstermin (Gruppensitzung oder Telefonat) teil.

Sieben Mütter und 4 Väter füllten die Fragebögen zur Erhebung des 6-Monats-Follow-ups trotz mehrmaliger Nachfrage nicht aus. Die Drop-out-Rate zu diesem Zeitpunkt betrug somit 22,6\%. Mütter, die nicht an der Nachuntersuchung teilnahmen, unterschieden sich hinsichtlich der Kurszufriedenheit bei Trainingsende nicht signifikant von Müttern, die die Fragebögen beantworteten.

\section{Zufriedenheit mit dem Triple P-Elterngruppentraining}

Im CSQ erreichten die Mütter im Mittel 76,2 (SD = 10,2) und die Väter 71,7 (SD = 11,1) Punkte. Die Mittelwerte der einzelnen Items sind getrennt für Mütter und Väter in Abbildung 1 dargestellt. Für alle Items lag die Bewertung im Mittel bei 5,86 ( $\mathrm{SD}=0,76)$ Punkten für die Mütter und bei 5,52 ( $\mathrm{SD}=$ 0,85 ) für die Väter (kein signifikanter Unterschied). Auch zeigte sich kein signifikanter Unterschied in der Zufriedenheit der Mütter in Abhängigkeit vom sozioökonomischen Status ( $U$ = $\left.135,0, \mathrm{~N}_{1}=20, \mathrm{~N}_{2}=18, \mathrm{p}=0,19 ; \mathrm{d}=0,38\right)$, vom Behandlungssetting $\left(\mathrm{U}=142,0, \mathrm{~N}_{1}=20, \mathrm{~N}_{2}=18, \mathrm{p}=0,27 ; \mathrm{d}=0,35\right)$ oder vom Vorliegen einer SSV $\left(\mathrm{U}=172,0, \mathrm{~N}_{1}=21, \mathrm{~N}_{2}=17, \mathrm{p}=0,85\right.$; $\mathrm{d}=0,08)$. Insgesamt bewerteten 100\% der Eltern die Qualität des Programms als gut bis hervorragend. 94\% waren mit der erhaltenen Unterstützung zufrieden bis sehr zufrieden. $92 \%$ gaben an, effektiver mit dem Verhalten ihres Kindes umgehen zu können, und 92\% schätzten das Verhalten ihres Kindes nach dem Training als etwas bis viel besser ein. $57 \%$ der Eltern hatten den Eindruck, ihre Partnerschaft habe von dem Programm profitiert, und $92 \%$ würden auf Triple $\mathrm{P}$ zurückkommen, wenn sie erneut erzieherische Hilfe benötigen würden.

\section{Anwendung der Triple P-Strategien im Alltag}

Zur Zeit der Nachuntersuchung lebten alle Kinder wieder bei ihren Eltern. Zu diesem Zeitpunkt wandten mehr als $90 \%$ der Mütter folgende Strategien «gelegentlich» bis «regelmäBig» an: «wertvolle Zeit», «mit dem Kind sprechen“, «Zuneigung zeigen», «beschreibend loben», «Aufmerksamkeit schenken», «Familienregeln», «direktes Ansprechen», «klare ruhige Anweisungen» und «logische Konsequenzen». Am seltensten kamen «stille Zeit» und «Auszeit» zum Einsatz. Ähnlich verhielt es sich mit den Vätern, die allerdings «wertvolle Zeit» und «Familienregeln» seltener anwendeten.

In Abhängigkeit vom sozioökonomischen Status der Familie ergaben sich keine signifikanten Unterschiede hinsichtlich der Anwendungshäufigkeit der einzelnen Triple P-Strategien im Alltag. Bei der Betrachtung des Behandlungssettings gaben hingegen die Mütter, deren Kind zu Kursbeginn (teil-) stationär behandelt wurde $(\mathrm{n}=13)$ an, signifikant häufiger «Fragen-Sagen-Tun» einzusetzen als Mütter, deren Kinder zu Kursbeginn ambulant behandelt wurden $(\mathrm{n}=18)(\mathrm{U}=68,0$; $\mathrm{p}<0,05 ; \mathrm{d}=0,80)$. Im Hinblick auf die ICD-10-Diagnose der Kinder wurden 2 Unterschiede signifikant: Mütter, deren Kinder die Kriterien für eine SSV $(n=13)$ erfüllten, gaben signifikant häufiger an, die «Punktekarte» $(U=66,0 ; p<0,05$; $\mathrm{d}=0,88)$ und die «Auszeit» anzuwenden $(\mathrm{U}=34,0, \mathrm{p}<0,001$; $\mathrm{d}=1,54)$ als Mütter, deren Kinder keine SSV aufwiesen $(\mathrm{n}=18)$. 


\section{Diskussion}

Obwohl das Triple P-Elterngruppentraining nicht für den Einsatz im Rahmen der kinderpsychiatrischen Behandlung entwickelt wurde, fanden sich in der vorliegenden Pilotstudie deutliche Hinweise für eine sehr gute Akzeptanz in diesem Setting. Die Drop-out-Rate zum Trainingsende (1,9\%) lag sogar deutlich niedriger als in der Präventionsstudie von Heinrichs und Mitarbeitern [2006] (11,3\%). Dieses Ergebnis ist angesichts des zumeist niedrigen sozioökonomischen Status der Eltern besonders bemerkenswert. Mehr noch, Mütter mit niedrigem sozioökonomischen Status waren in der vorliegenden Studie von der Tendenz her sogar etwa zufriedener mit dem Elterntraining als die übrigen Mütter $(\mathrm{d}=0,39)$. Keine signifikanten Unterschiede in der Zufriedenheit der Mütter fanden sich hingegen in Abhängigkeit vom Behandlungssetting (ambulant vs. (teil-)stationär) und dem Vorliegen einer SSV (SSV vs. keine SSV). Aufgrund der geringen Stichprobengröße wurde in der vorliegenden Pilotstudie auf weitere differenzielle Analysen verzichtet.

Insgesamt waren die Zufriedenheitsraten der Teilnehmer der aktuellen Studie im CSQ ähnlich hoch wie in 2 Präventionsstudien im deutschen Sprachraum [Cina et al., 2006; Heinrichs et al., 2006]. So waren z.B. in der Studie von Cina et al. [2006], in die 1290 Eltern eingeschlossen werden konnten, 93\% der Mütter und Väter zufrieden bis sehr zufrieden mit dem Programm, 95\% bewerteten die Qualität des Trainings als gut bis hervorragend, und $97 \%$ gaben an, dass sie auf Triple $\mathrm{P}$ zurückkommen würden, wenn sie erneut erzieherische Probleme hätten. Da in der vorliegenden Studie allerdings deutlich mehr Zeit als üblich für die Vermittlung der Triple P-Strategien verwendet wurde, ist unklar, wie die Zufriedenheit unter Standardbedingungen des Elterntrainings gewesen wäre. Die teilnehmenden Väter zeigten im Mittel fast durchgängig eine geringere Zufriedenheit mit dem Training als die Mütter, allerdings war diese Differenz statistisch nicht signifikant.

Die Ergebnisse zur Akzeptanz der Kursinhalte, gemessen über die Anwendungshäufigkeit der vermittelten Strategien 6 Monate nach Kursende, waren bei Vätern und Müttern ähnlich. Auch fanden sich keine signifikanten Unterschiede in Abhängigkeit vom sozioökonomischen Status. Dass die Strategien «stille Zeit» und «Auszeit» insgesamt am seltensten angewandt wurden, verwundert nicht, da sie gemäß Manual auch nur bei größerem Problemverhalten eingesetzt werden sollten. Passend dazu wandten allerdings Mütter, deren Kinder die Kriterien für eine SSV erfüllten, signifikant häufiger die «Auszeit» an (und auch die komplexe Strategie «Punktekarte») als Mütter, deren Kinder keine SSV aufwiesen.

Auch in der Studie von Cina und Kollegen [2006] schätzten mindestens $90 \%$ der Teilnehmer 6 Monate nach Kursende die folgenden Strategien als hilfreich ein: «wertvolle Zeit», «mit dem Kind sprechen», «Zuneigung zeigen», «beschreibendes Loben», «Aufmerksamkeit schenken», «beiläufiges Lernen», «direktes Ansprechen», «klare ruhige Anweisungen» und «logische Konsequenzen». «Stiller Stuhl» und «Punktekarte» wurden als am wenigsten hilfreich erlebt [Cina et al., 2006]. $\mathrm{Zu}$ ähnlichen Ergebnissen kam auch von Wulfen [2007].

Die vorliegende Pilotstudie hat einige methodische Einschränkungen. So wurde die Indikation für eine Teilnahme am Elterngruppentraining nicht genauer operationalisiert, sondern blieb dem klinischen Eindruck bzw. der Erfahrung des jeweiligen Fallführers überlassen. Außerdem wurde nicht systematisch erfasst, in wie vielen Fällen die Teilnahme am Triple P-Elterngruppentraining empfohlen wurde, sie wurde aber sicherlich bei Weitem nicht allen 173 Kindern angeboten. Wie oft Eltern dieser Empfehlung nicht nachkamen bzw. aus welchen Gründen dies geschah, wurde ebenfalls nicht genau erfasst.

Inwieweit sich durch die Kursteilnahme Veränderungen im Erziehungsverhalten bzw. in der Eltern-Kind-Interaktion ergaben, konnte nicht durch objektive Verfahren wie standardisierte Verhaltensbeobachtungen erfasst werden, da dies den Rahmen einer Pilotstudie gesprengt hätte. Stattdessen wurde die subjektive Sicht der teilnehmenden Eltern erfragt, wobei allerdings in keinem der eingesetzten Fragebogenverfahren auf sozial erwünschtes Verhalten kontrolliert wurde. Bei allen Eltern, insbesondere aber bei Eltern von (teil-)stationären Patienten, könnte sich eine positive Erwartungshaltung bezüglich der Wirksamkeit entwickelt haben, da Triple $\mathrm{P}$ als pädagogisches Grundkonzept in der gesamten Klinik Anwendung findet.

$\mathrm{Zu}$ guter Letzt ist die Drop-out-Rate von 22,6\% bei Befragung 6 Monate nach dem Training zu nennen. Da sich die Kurszufriedenheit von Müttern, die nicht an der Nachuntersuchung teilnahmen, und solchen, die die Fragebögen beantworteten, nicht signifikant unterschied, dürfte jedoch zumindest kein systematischer Fehler vorgelegen haben.

Durch die genannten Einschränkungen ist eine Generalisierbarkeit der Ergebnisse nur bedingt möglich, weshalb die Anwendung an einer größeren multizentrischen Stichprobe sinnvoll wäre, in der auch objektive Beurteilungsverfahren Einsatz finden sollten. Differenzierte Analysen der Variablen sozioökonomischer Status, Behandlungssetting und ICD-10Diagnose könnten letztlich auch spezifischere Aussagen darüber erlauben, welche Eltern wie gut von einem Triple P-Elterngruppentraining profitieren.

Zusammenfassend kann dennoch festgestellt werden, dass die Ergebnisse der vorliegenden Pilotuntersuchung zur Akzeptanz eines vom Zeitumfang her adaptierten Triple P-Elterngruppentrainings im Rahmen der kinderpsychiatrischen Behandlung sehr vielversprechend sind, insbesondere auch im Hinblick auf Familien mit niedrigem sozioökonomischen Status.

\section{Disclosure Statement}

Die Autoren erklären hiermit, dass keine Interessenskonflikte bezüglich des Manuskripts bestehen. 


\section{Literatur}

Bachmann M, Bachmann C, Rief W, Mattejat F: Wirksamkeit psychiatrischer und psychotherapeutischer Behandlungen bei psychischen Störungen von Kindern und Jugendlichen - Teil II: ADHS und Störung des Sozialverhaltens. Z Kinder Jugendpsychiatr Psychother 2008;36:321-333.

Cina A, Bodenmann G, Hahlweg K, Discherl T, Sanders MT: Triple P: Theoretischer und empirischer Hintergrund und erste Erfahrungen im deutschsprachigen Raum. Zeitschrift für Familienforschung 2006;1:66-88.

Cohen J: Statistical Power Analysis for the Behavioral Sciences. Hillsdale, Erlbaum, 1988.

Deutsche Gesellschaft für Kinder- und Jugendpsychiatrie und Psychotherapie, Bundesarbeitsgemeinschaft Leitender Klinikärzte für Kinder- und Jugendpsychiatrie und Psychotherapie, Berufsverband der Ärzte für Kinder- und Jugendpsychiatrie und Psychotherapie (eds): Leitlinien zu Diagnostik und Therapie von psychischen Störungen im Säuglings-, Kindes- und Jugendalter. Köln, Deutscher Ärzte-Verlag, 2007.

Döpfner M, Berner W, Flechtner H, Lehmkuhl G, Steinhausen HC: Psychopathologisches BefundSystem für Kinder und Jugendliche (CASCAP-D). Göttingen, Hogrefe, 1999.

Döpfner M, Görtz-Dorten A, Lehmkuhl G: DISYPSII. Diagnostik-System für psychische Störungen nach ICD-10 und DSM-IV für Kinder und Jugendliche - II. Manual. Bern, Hans Huber, 2008.

Eyberg SM, Nelson MM, Boggs SR: Evidence-based psychosocial treatments for children and adolescents with disruptive behavior. J Clin Child and Adolesc Psychol 2008;37:215-237.
Heinrichs N, Hahlweg K, Kuschel A, Krüger S, Bertram H, Harstick S, Naumann S: Triple P aus der Sicht der Eltern: Teilnahmeraten und Kurszufriedenheit in Abhängigkeit von soziodemographischen Charakteristika und Migration. Kindheit und Entwicklung 2006;15:19-26.

Krajewski K, Liehm S, Schneider W: DEMAT 2+. Deutscher Mathematiktest für zweite Klassen. Göttingen, Beltz Test GmbH, 2004.

Lange M, Kamtsiuris P, Lange C, Schaffrath Rosario A, Stolzenberg H, Lampert T: Messung soziodemographischer Merkmale im Kinder- und Jugendgesundheitssurvey (KiGGS) und ihre Bedeutung am Beispiel der Einschätzung des allgemeinen Gesundheitszustands. Bundesgesundheitsblatt Gesundheitsforschung Gesundheitsschutz 2007;50:578-589.

Linder M, Grissemann H: Zürcher Lesetest, ZLT. Manual. Bern, Hans Huber, 2000.

Markie-Dadds C, Turner KMT, Sanders MR: Das Triple P Gruppenarbeitsbuch. Das Begleitbuch zum Triple P-Gruppentraining, ed 2. Münster, PAG Institut für Psychologie AG, 2007.

Müller R: DRT 2. Diagnostischer Rechtschreibtest für 2. Klassen, ed 4. Göttingen, Beltz Test GmbH, 2004.

Petermann F, Petermann U (eds): Hamburg-WechslerIntelligenztest für Kinder IV, ed 2. Bern, Hans Huber, 2008.

Ricken G, Fritz A, Schuck KD, Preuß U (eds): HAWIVA-III, Hannover-Wechsler-Intelligenztest für das Vorschulalter-III, Manual zur Durchführung und Auswertung. Göttingen, Hans-Huber, 2007.
Robert-Koch-Institut und Bundeszentrale für gesundheitliche Aufklärung (eds): Erkennen - Bewerten Handeln: Zur Gesundheit von Kindern und Jugendlichen in Deutschland. Berlin, Robert-Koch-Institut, 2008.

Rustenbach SJ: Metaanalyse. Eine anwendungsorientierte Einführung. Bern, Huber, 2003.

Sanders MR: Development, evaluation, multinational dissemination of the Triple P-positive parenting program. Annu Rev Clin Psychol 2012;8:345-379.

Stadler C: Störungen des Sozialverhaltens. Sind neue Erklärungsansätze eine Grundlage für eine evidenzbasierte Klassifikation und Behandlung? Z Kinder Jugendpsychiatr Psychother 2012;40:7-19.

Tewes U, Rossmann P, Schallberger U: HAWIK-III Hamburg-Wechsler-Intelligenztest für Kinder Dritte Auflage; Manual. Bern, Hans Huber, 2002.

Turner KMT, Markie-Dadds C, Sanders MR: Trainermanual für das Triple P Gruppenprogramm. Münster, PAG Institut für Psychologie, 2002.

von Wulfen Y: Wen und was erreicht Prävention in der Praxis? Dissemination und Effektivität des Triple P-Elterngruppentrainings im Beratungsalltag in Deutschland. Münster, Verlag für Psychotherapie, 2007.

Zimmermann P, Fimm B: TAP. Testbatterie zur Aufmerksamkeitsprüfung. Version 2,1. Herzogenrath, Vera Fimm Psychologische Testsysteme, 2007. 